\title{
Niche astrocytes promote the survival, proliferation and neuronal differentiation of co-transplanted neural stem cells following ischemic stroke in rats
}

\author{
LI LUO $^{1-3^{*}}$, KAIHUA GUO ${ }^{2 *}$, WENGUO FAN ${ }^{1}$, YINGHONG LU $^{2}$, LIZHI CHEN $^{2}$, \\ YANG WANG $^{2}$, YIJIA SHAO ${ }^{2}$, GONGXIONG WU ${ }^{4}$, JIE XU ${ }^{2}$ and LANHAI LÜ ${ }^{1}$
}

\begin{abstract}
${ }^{1}$ Guanghua School of Stomatology, Guangdong Provincial Key Laboratory of Stomatology and Institute of Stomatological Research, Hospital of Stomatology, Sun Yat-sen University, Guangzhou, Guangdong 510055; ${ }^{2}$ Department of Anatomy and Neurobiology, Zhongshan School of Medicine, Sun Yat-sen University, Guangzhou, Guangdong 510080;

${ }^{3}$ Department of Anatomy, School of Basic Medicine, Guangdong Pharmaceutical University, Guangzhou, Guangdong 510006, P.R. China; ${ }^{4}$ Research Division, Joslin Diabetes Center, Harvard Medical School, Boston, MA 02215, USA
\end{abstract}

Received September 22, 2015; Accepted June 15, 2016

DOI: $10.3892 /$ etm.2016.4016

\begin{abstract}
Niche astrocytes have been reported to promote neuronal differentiation through juxtacrine signaling. However, the effects of astrocytes on neuronal differentiation following ischemic stroke are not fully understood. In the present study, transplanted astrocytes and neural stem cells (NSCs) were transplanted into the ischemic striatum of transient middle cerebral artery occlusion (MCAO) model rats $48 \mathrm{~h}$ following surgery. It was observed that the co-transplantation of astrocytes and NSCs resulted in a higher ratio of survival and proliferation of the transplanted NSCs, and neuronal differentiation, in MCAO rats compared with NSC transplantation alone. These results demonstrate that the co-administration of astrocytes promotes the survival and
\end{abstract}

Correspondence to: Professor Jie Xu, Department of Anatomy and Neurobiology, Zhongshan School of Medicine, Sun Yat-sen University, 74 Zhongshan Er Road, Guangzhou, Guangdong 510080, P.R. China

E-mail:xujie@mail.sysu.edu.cn

Dr Lanhai Lü, Guanghua School of Stomatology, Guangdong Provincial Key Laboratory of Stomatology and Institute of Stomatological Research, Hospital of Stomatology, Sun Yat-sen University, 56 LingYuanXi Road, Guangzhou, Guangdong 510055, P.R. China

E-mail: 1vlanhai@mail.sysu.edu.cn

\section{*Contributed equally}

Abbreviations: NSCs, neural stem cells; AS, astrocytes; MCAO, middle cerebral artery occlusion; BrdU, bromodeoxyuridine; P0, postnatal day 0; eGFP, enhanced green fluorescent protein; SVZ, subventricular zone; SGZ, subgranular zone

Key words: niche astrocyte, neural stem cells, middle cerebral artery occlusion, ischemia, cell transplantation, stem cell therapy neuronal differentiation of NSCs in the ischemic brain. These results suggest that the co-transplantation of astrocytes and NSCs is more effective than NSCs alone in the production of neurons following ischemic stroke in rats.

\section{Introduction}

Strokes, a leading cause of death and disability worldwide, cause a disturbance of neuronal circuitry and disruption of the blood-brain barrier that can lead to functional disabilities (1). Currently, there is no pharmacological intervention that can effectively improve neurological outcome in patients with stroke (2). While conventional therapeutic strategies focus on preventing brain damage, stem cell transplantation has the potential to repair the injured brain tissue. Recently, neural stem cells (NSCs) have been developed and used in preclinical studies as therapy for stroke $(3,4)$.

NSCs are undifferentiated neural cells that are multipotent and possess the capacity for self-renewal. NSCs differentiate into three cell lineages, neurons, astrocytes and oligodendrocytes $(5,6)$. An increasing number of studies highlight the potential of NSC transplantation as a novel therapeutic approach for stroke (7-9). NSCs are primarily produced in the subventricular zone (SVZ) of the lateral ventricles and the subgranular zone (SGZ) of the hippocampus in the adult nervous system (10). Previous studies investigating the association between NSCs and ectogenic and endogenic factors have increased the understanding of how to modulate the proliferation and differentiation of NSCs during neurogenesis $(11,12)$. NSCs reside in specialized microenvironments, or 'niches', that regulate their self-renewal and differentiation activities (13). For example, the continuous production of neuroblasts is maintained by the SVZ microenvironmental niche $(3,4)$. It is widely believed that enhancing the neurogenic activities of endogenous NSCs will provide a much-needed therapeutic option for brain injuries (14). Astrocytes are typically considered cellular components of these stem cell niches, and astrocytes isolated from postnatal 
day 0 (P0) mouse brains or adult SGZs are referred to as niche astrocytes (NAs) (15). NAs isolated from neonatal mouse brains show increased NSC proliferation and promote neuronal differentiation (15). In addition, a previous study demonstrated that astrocytes could enhance the capacity of NSCs to differentiate into neurons under co-culture conditions in vitro (16). NSCs transplanted together with NAs may be a novel strategy for the treatment of neural injury. In the present study, the effect of NAs on the regulation of co-transplanted NCSs was evaluated in rat models following transient cerebral ischemia.

\section{Materials and methods}

Animal surgery. Twenty-four healthy adult male

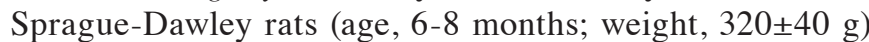
were purchased from the Laboratory Animal Center at Zhongshan Medical School (Sun Yat-sen University, Guangzhou, China). Rats were kept in an environment with a $12 \mathrm{~h} \mathrm{light/dark}$ cycle at $20 \pm 3^{\circ} \mathrm{C}$ and had ad libitum access to food and water. Experimental protocols used in the present study were performed in accordance with the guidelines of the Experimental Laboratory Animal Committee of Sun Yat-sen University Health Science Center (Guangzhou, China) and the National Institutes of Health's Guide for the Care and Use of Laboratory Animals (17). Rats were anesthetized with the intraperitoneal injection of chloral hydrate (400 mg/kg body weight; Shanghai Bang Jing Industrial Co., Ltd., Shanghai, China; cat. no. 34883-39-1) and subjected to right middle cerebral artery occlusion (MCAO) surgery to induce focal cerebral infarction by insertion of a 4-0 nylon suture. Following $1 \mathrm{~h}$, the nylon suture was withdrawn for reperfusion. To label the dividing cells, bromodeoxyuridine (BrdU; Sigma Aldrich, St. Louis, MO, USA) was intraperitoneally administered (50 $\mathrm{mg} / \mathrm{kg}$ body weight) following transplantation.

Cell culture. Enhanced green fluorescent protein positive $\left(\mathrm{eGFP}^{+}\right)$NSCs were obtained from the SVZ of neonatal C57BL/6-eGFP ${ }^{+}$mice (1 day old) provided by Cyagen Biosciences Inc. (Guangzhou, China). The cells were seeded into $25 \mathrm{~cm}^{2}$ culture flasks (Corning Incorporated, NY, USA) at a density of $5 \times 10^{5}$ cells $/ \mathrm{ml}$ in serum-free culture medium containing Dulbecco's modified Eagle's medium (DMEM; 1:1; Gibco; Thermo Fisher Scientific, Inc., Beijing, China). This was supplemented with $1 \%$ B-27, $1 \% \mathrm{~N}-2,20 \mathrm{ng} / \mathrm{ml}$ epidermal growth factor, $20 \mathrm{ng} / \mathrm{ml}$ basic fibroblast growth factor (all Invitrogen; Thermo Fisher Scientific, Inc.), penicillin (100 units $/ \mathrm{ml})$ and streptomycin $(0.1 \mathrm{mg} / \mathrm{ml})$. Cultured NSCs aggregated into free-floating neurospheres and were passaged (split ratio, 1:4) by mechanical dissociation between every 6 and 7 days.

For astrocyte primary cultures, astrocytes were extracted from the cerebral cortex of P0 C57BL/6-eGFP-mice. Cells were seeded at a density of $5 \times 10^{5}$ cells $/ \mathrm{ml}$ on poly-L-lysine-treated $25 \mathrm{~cm}^{2}$ culture flasks with DMEM (1:1), supplemented with $10 \%$ fetal bovine serum (Gibco; Thermo Fischer Scientific, Inc.), 100 units/ml penicillin and $100 \mu \mathrm{g} / \mathrm{ml}$ streptomycin. The cells were fed with fresh media every 2-3 days and subcultured (passage ratio, 1:4) weekly. To determine the purity of the astrocytes they were labeled with nuclear dye Hoechst 33342 (20 ng/ml; Biotium, Hayward, CA, USA) and immunofluorescence staining for glial fibrillary acidic protein (GFAP) was performed (described below), in order to acquire the percentage of GFAP-positive cells among the Hoechst 33342-labeled cells. The purity of the astrocytes used for implantation in the current study was $\geq 95 \%$.

Cell transplantation. Cultured NSCs and astrocytes used in the study were passaged three times prior to implantation. On the day of transplantation, NSCs and astrocytes were centrifuged at $250 \mathrm{x} g$ for $5 \mathrm{~min}$ at $4^{\circ} \mathrm{C}$ and resuspended in PBS, yielding $2 \times 10^{4}$ viable cells $/ \mu 1$. The NSC and astrocyte suspension was kept on ice throughout the surgery. MCAO rats were assigned into the following two groups (each $n=12$ ): One group was transplanted with NSCs and astrocytes; and one group transplanted with NSCs. Forty-eight h following MCAO, the cerebral ischemic rats were anesthetized with chloral hydrate and placed in a stereotactic instrument (Narishige International, Ltd., London, UK). Next, the cells were injected into the right striatum of the ischemic cerebral hemisphere (Fig. 1A) of the MCAO rats $(0.5 \mathrm{~mm}$ anterior and $3 \mathrm{~mm}$ lateral from the bregma, $5 \mathrm{~mm}$ from the brain surface) using a microcrystal line syringe at a speed of $0.5 \mu \mathrm{l} / \mathrm{min}$, with a $5 \mathrm{~min}$ delay prior to removal of the syringe. All surgical procedures were performed under aseptic conditions. Following cell delivery, all rats were administered a subcutaneous injection of cyclosporine A $(10 \mathrm{mg} / \mathrm{kg}$ body weight; Beyotime Institute of Biotechnology, Shanghai, China) every other day. The survival of transplanted NSCs in MCAO rats was investigated 7 days following transplantation, and the proliferation and differentiation of transplanted NSCs was assessed 14 days following transplantation.

Immunofluorescence staining. Rats were sacrificed with a peritoneal injection of $10 \%$ chloral hydrate (Sigma-Aldrich), transcardially perfused with saline followed by ice-cold $4 \%$ paraformaldehyde (Wuhan Boster Biological Technology, Ltd., Wuhan, China) a for $24 \mathrm{~h}$ at $4^{\circ} \mathrm{C}$. Coronal sections of $30 \mu \mathrm{m}$ were cut and processed for immunostaining, as described previously (18). Triple-label immunofluorescence staining was performed on the sections. Following blocking, using $10 \%$ normal goat serum (Beyotime Institute of Biotechnology) for $1 \mathrm{~h}$ at room temperature, the following primary antibodies were added: Mouse anti-GFAP, a marker for mature astrocytes (1:500; Abcam, Cambridge, UK; cat. no. ab3670); rabbit anti-oligodendrocyte (Oli) to label oligodendrocytes (1:500, Abcam; cat no. ab53041); rabbit anti-doublecortin (Dcx) to label migrating neuroblasts (1:1,000; Cell Signaling Technology, Inc., Beverly, MA, USA; cat. no. 4604); mouse anti-neuronal nuclei (NeuN) to label mature neurons (1:500; EMD Millipore, Billerica, MA, USA; cat. no. MAB377); mouse anti-BrdU to label dividing cells (1:500; Sigma-Aldrich; cat. no. B8434); mouse anti-microtubule-associated protein-2 (MAP-2; 1:500; Sigma-Aldrich; cat. no. M4403); mouse anti-choline acetyltransferase (ChAT; 1:500; Sigma-Aldrich; cat. no. AMAB91129); mouse anti-progranulin (PGRN; 1:200; R\&D Systems, Inc., Minneapolis, MN, USA; cat. no. AF2557); mouse 
A

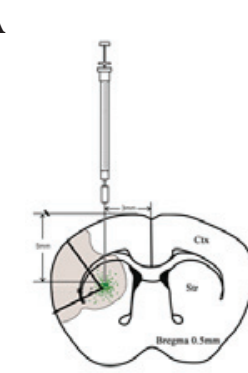

D
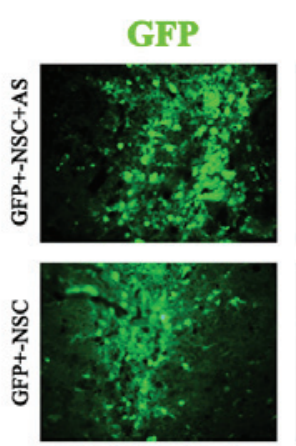

B

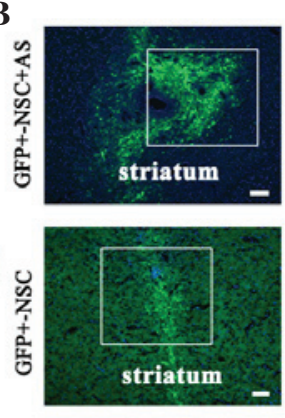

BrdU
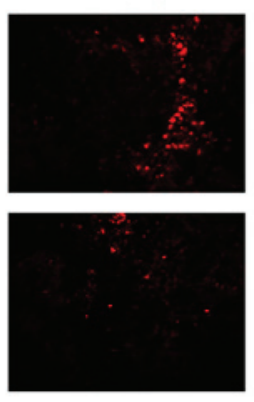
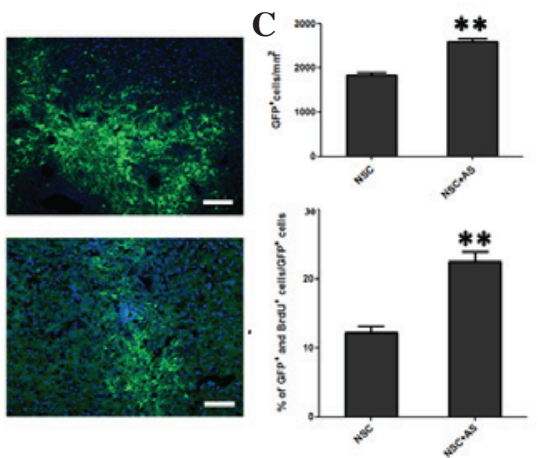

Hoe

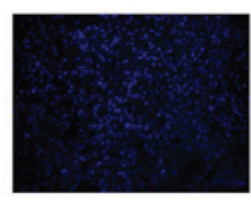

Merge
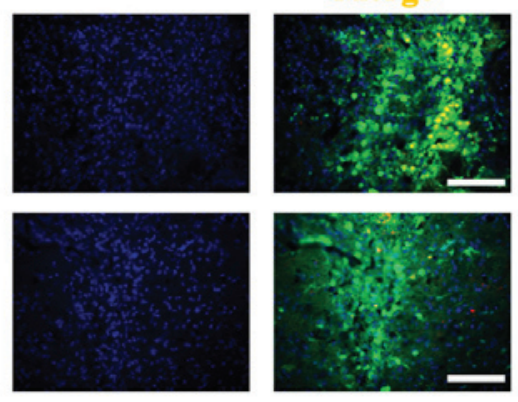

Figure 1. Astrocytes promote the survival and proliferation of transplanted NSCs in MCAO rats. (A) Schematic drawing of a frontal section through the striatum, illustrating the distribution and dispersion of $\mathrm{GFP}^{+}$grafted cells. The shaded area indicates the region of ischemia. (B) On day 7 following transplantation, GFP NSCs were observed in rats from the co-transplantation group and NSC alone group. (C) A significantly increased number of living cells were observed in rats co-transplanted with AS compared with rats without $\mathrm{AS} ;{ }^{* *} \mathrm{P}<0.01$. (D) BrdU incorporation assays showed that GFP BrdU ${ }^{+}$cells were increased in the co-transplantation group compared with the group without astrocytes. Scale bars, $100 \mu \mathrm{m}$. GFP, green fluorescent protein; NSC, neural stem cell; AS, astrocytes; Ctx, cortex; Str, striatum; BrdU, bromodeoxyuridine; Hoe, Hoechst 33342.

anti-post-synaptic density protein 95 (PSD-95; 1:200; BIOSS, Beijing, China; cat. no. bs-0179R); mouse anti-gamma-aminobutyric acid (GABA; 1:200; BIOSS; cat. no. bs-2252) and mouse anti-synapsin-1 (1:200; BIOSS; cat. no. bs-3501R). Sections were incubated overnight at $4^{\circ} \mathrm{C}$. Following three washes with PBS (10 min/wash), sections were incubated with the appropriate fluorophore-conjugated secondary antibody [Alexa Fluor 594 goat anti-mouse (cat. no. R37121), Alexa Fluor 594 donkey anti-rat (cat. no. A-21209), Alexa Fluor 594 goat anti-rabbit (cat. no. R37177) and Alexa Fluor 405 goat anti-sheep (cat. no. 35500BID) (all Invitrogen; Thermo Fisher Scientific, Inc.)] for $1 \mathrm{~h}$ at room temperature. Nuclei were stained for $5 \mathrm{~min}$ with Hoechst $33342(20 \mathrm{ng} / \mathrm{ml}$, Biotium Inc. Hayward, CA). A protocol-naive individual then captured images of the sections at a magnification of x200 using a fluorescence microscope (BX51WI; Olympus Corporation, Tokyo, Japan). Then, the number of cells in 10 randomly selected images was counted. The percentage of $\mathrm{eGFP}^{+}, \mathrm{BrdU}^{+}$and $\mathrm{NeuN}^{+}$cells per image field out of the total number of cells in each independent experiment was calculated. All experiments were performed three times.

Statistical analysis. The results are expressed as the mean \pm the standard error of the mean. Statistical comparisons between groups were performed with one-way analysis of variance, followed by a Student's t-test using SPSS software (version 17.0; SPSS, Inc., Chicago, IL, USA). P<0.05 was considered to indicate a statistically significant difference.

\section{Results}

Niche astrocytes promote the survival and proliferation of transplanted NSCs in MCAO rats. To study the effect of astrocytes on the survival of transplanted NSCs in MCAO rats, the transplanted NSCs were evaluated 7 days after transplantation. $\mathrm{eGFP}^{+}$cells were observed in rats that received NSCs with and without astrocytes (Fig. 1B). The number of $\mathrm{GFP}^{+} \mathrm{NSCs}$ was significantly higher in the co-transplantation group, compared with the group receiving NSCs alone $\left(2,588 \pm 76.88\right.$ cells $/ \mathrm{mm}^{2}$ vs. $1,836 \pm 57.41$ cells $/ \mathrm{mm}^{2} ; \mathrm{P}<0.01$; Fig. 1C). In the co-transplantation group an increased number of transplanted $\mathrm{GFP}^{+} \mathrm{NSCs}$ were $\mathrm{BrdU}^{+}$(Fig. 1D). The ratio of $\mathrm{BrdU}^{+} / \mathrm{GFP}^{+}$cells to $\mathrm{BrdU}-/ \mathrm{GFP}^{+}$cells was significantly increased in the presence of astrocytes $(22.40 \pm 1.50 \%$ vs. $12.20 \pm 0.93 \%$; $\mathrm{P}<0.01$; Fig. 1C). These results suggest that NAs promote the proliferation of transplanted NSCs in vivo.

Niche astrocytes promote neuronal differentiation of transplanted NSCs in MCAO rats. To study the effect of astrocytes on the differentiation of transplanted NSCs in MCAO rats, the fate of $\mathrm{GFP}^{+}$cells was investigated 14 days after cell transplantation. The results showed that transplanted NSCs could differentiate into $\mathrm{Dcx}^{+}, \beta I I I-\mathrm{Tubulin}^{+}, \mathrm{NeuN}^{+}$, $\mathrm{GFAP}^{+}$and oligodendrocyte ${ }^{+}$cells (Fig. 2A). On day 14, $\mathrm{GFP}^{+} / \mathrm{NeuN}^{+}$cells were observed in the striatum (Fig. 2B). The ratio of $\mathrm{NeuN}^{+}$cells in the population of $\mathrm{GFP}^{+}$cells was evaluated, showing that there was a significantly higher level of neuronal differentiation in co-transplanted cells compared 
A
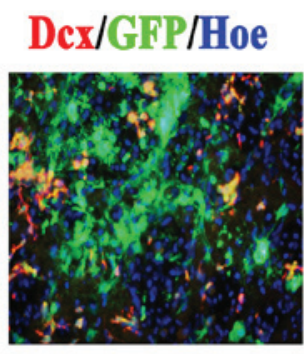

Tuj/GFP/Hoe

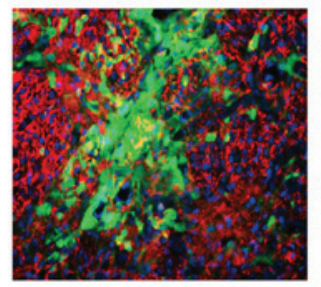

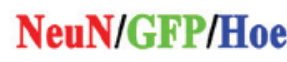

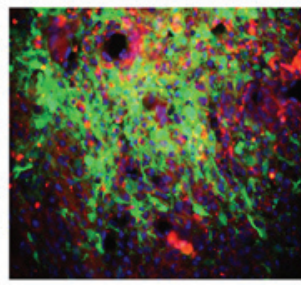

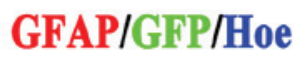

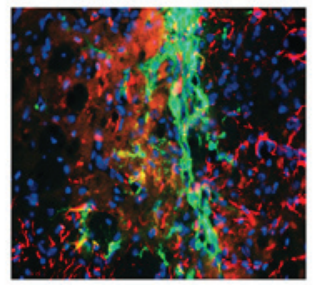

\section{Oli/GFP/Hoe}

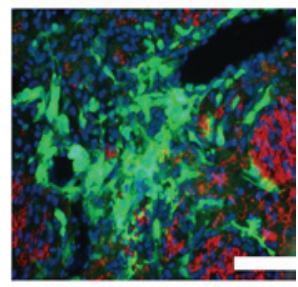

B

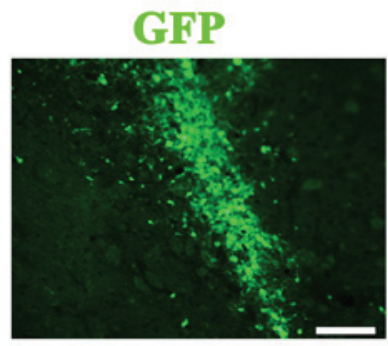

C

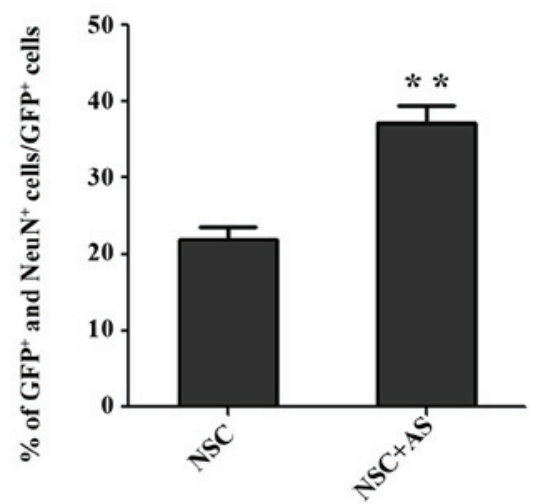

NeuN
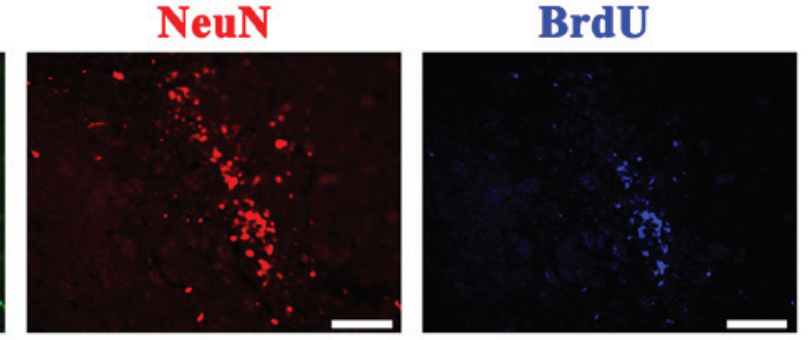

D
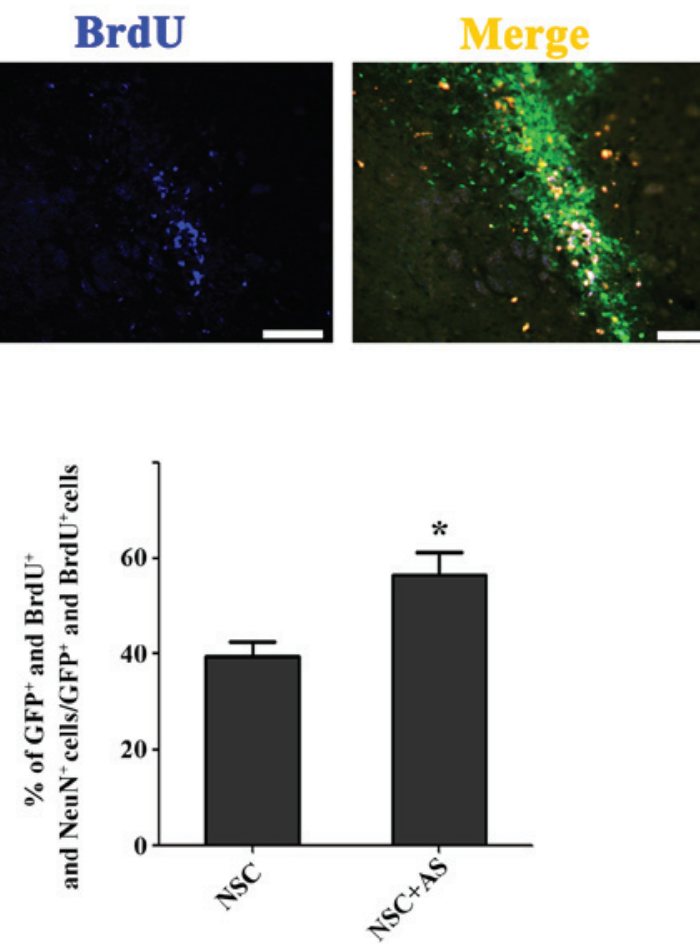

Figure 2. Astrocytes promote neuronal differentiation of transplanted NSCs in MCAO rats. (A) On day 14 after cell transplantation, transplanted NSCs could differentiate into Dcx, $\beta$ III-Tubulin (Tuj), NeuN, GFAP and Oli positive cells. (B) GFP $\mathrm{NeuN}^{+} \mathrm{BrdU}^{+}$cells were observed 14 days after cell transplantation. (C) Quantitative analysis showed the proportion of $\mathrm{GFP}^{+} \mathrm{NeuN}^{+}$cells to $\mathrm{GFP}^{+}$cells was significantly higher in the NSC+AS group compared with the NSC alone group; ${ }^{* *} \mathrm{P}<0.01$. (D) The percentage of $\mathrm{GFP}^{+} \mathrm{NeuN}^{+} \mathrm{BrdU}^{+}$cells in the population of $\mathrm{GFP}^{+} \mathrm{BrdU}^{+}$cells was significantly higher in the brains of rats subjected to co-transplantation compared with those receiving NSCs alone; ${ }^{*} \mathrm{P}<0.05$. Scale bars, $100 \mu \mathrm{m}$. Dcx, doublecourtin; GFP, green fluorescent protein; Hoe, Hoechst 33342; NeuN, neuronal nuclei; GFAP, glial fibrillary acidic protein; Oli, oligodendrocyte; BrdU, bromodeoxyuridine.

with cells transplanted with NSCs alone $(37.13 \pm 2.30 \%$ vs. $21.67 \pm 1.72 \%$; $\mathrm{P}<0.01$; Fig. 2C). The fate of proliferative $\mathrm{GFP}^{+}$ cells $\left(\mathrm{GFP}^{+} / \mathrm{BrdU}^{+}\right)$was also analyzed. The percentage of $\mathrm{GFP}^{+} / \mathrm{NeuN}^{\mathrm{v}} \mathrm{s} . \mathrm{BrdU}^{+}$cells in the population of $\mathrm{GFP}^{+} / \mathrm{BrdU}^{+}$ cells was significantly higher in the co-transplantation group compared with the group receiving NSCs alone $(56.53 \pm 4.82 \%$ vs. 39.47士3.20\%; $\mathrm{P}<0.05$; Fig. 2D). These results indicate that co-transplantation of astrocytes promotes neuronal differentiation of transplanted NSCs in MCAO rats.

Grafted NSCs express functional proteins. To confirm whether the neonatal neurons from NSCs could help reconstruct synapses in the border of the infarction, the detection of neuronal marker proteins was performed. Results showed a subpopulation of transplanted NSCs at the lesion boundary and number of migrating cells that could express Synapsin-1, GABA, PSD-95, MAP-2, ChAT and PGRN proteins (Fig. 3).

\section{Discussion}

NSC transplantation therapy for strokes is promising $(7,8)$. NAs, as a cellular component of the stem cell microenvironment, are reported to display regional heterogeneity in their ability to regulate neurogenesis (19). In the present study, it is demonstrated that co-transplantation of NAs promotes the survival, proliferation and neuronal differentiation of SVZ-derived NSCs in the ischemic brain.

Previous studies have reported that numerous factors can influence the efficiency of stem cell transplantation therapy $(20,21)$. Consistent with previous studies, the data in the current study shows that transplantation of NSCs together with astrocytes results in markedly enhanced survival, proliferation and neuronal differentiation of NSCs, which indicates that astrocytes serve a critical role in modulating NSC fate determination $(22,23)$. Astrocytes can secrete neurotrophins 

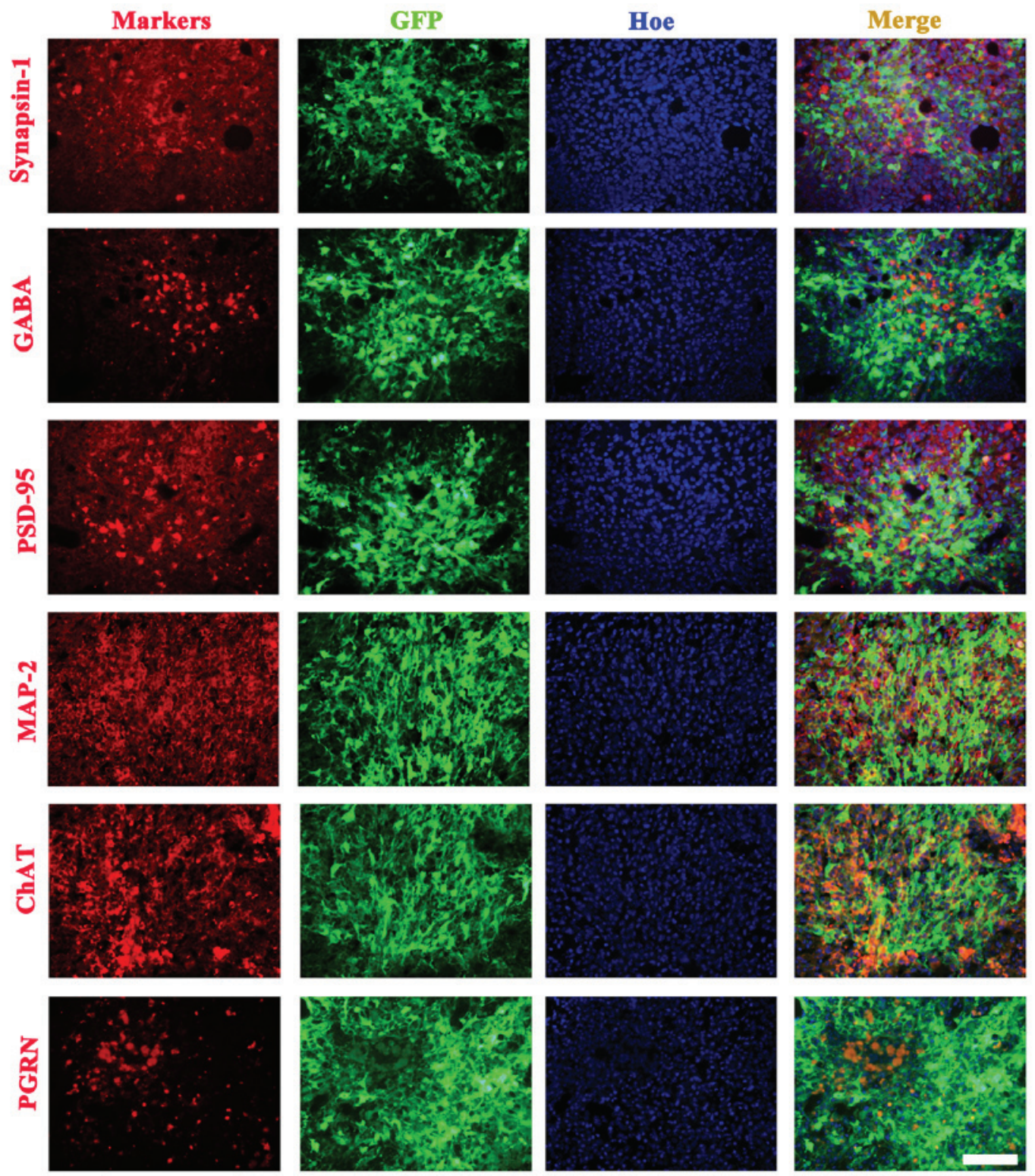

Figure 3. Subpopulations of transplanted NSCs express functional neuronal proteins 7 days after cell transplantation. Grafted NSCs expressed Synapsin-1, GABA, PSD-95, MAP-2, ChAT and PGRN. Scale bars, $100 \mu \mathrm{m}$. GFP, green fluorescent protein; Hoe, Hoechst 33342; PSD-95, postsynaptic density protein-95; MAP-2, microtubule-associated protein-2; ChAT, choline acetyltransferase; PGRN, progranulin; GABA, $\gamma$-aminobutyric acid.

(nerve growth factor, ciliary neurotrophic factor, brain-derived neutrotrophic factor and neurotrophin-3), chemokines (interleukins -4 and -6) and cytokines (leukemia inhibitory factor) under different conditions, through which it can regulate the proliferation, differentiation and migration of NSCs (24). However, the exact signaling pathways through which astrocytes regulate the behavior of NSCs in co-transplantation conditions remains unknown. Further studies will be performed to investigate the molecular mechanism underlying the synergistic function between astrocytes and NSCs during cell transplant therapy.

Nedachi et al (25) reported that PGRN could enhance neural progenitor cell proliferation. The results in the present study showed that PGRN was expressed in a number of subpopulations of grafted NSCs on day 7 and 14 following transplantation. Previous results have indicated that PGRN is abundantly expressed in neurospheres and their differentiated cell lineages (5). In the current study, antibodies against Synapsin-1, GABA, PSD-95, MAP-2 and ChAT were used, from which the results indicated that the differentiated cell lineages from grafted NSCs were involved in the ischemic brain repair.

In conclusion, the results in the present study demonstrate that NAs serve a synergistic role to promote the survival, proliferation and neuronal differentiation of NSCs in the ischemic brain. NSC transplantation together with NAs may be a novel strategy for the repair of stroke injury.

\section{Acknowledgements}

The present study was supported by The National Natural Science Foundation of China for Youth (grant no. 81000562), 
the Fundamental Research Funds for the Central Universities (grant no. 14ykpy33) and Medical Scientific Research Foundation of Guangdong Province, China (grant no. A2015131).

\section{References}

1. Ding DC, Lin CH, Shyu WC and Lin SZ: Neural stem cells and stroke. Cell Transplant 22: 619-630, 2013.

2. Ding DC, Shyu WC, Lin SZ and Li H: Current concepts in adult stem cell therapy for stroke. Curr Med Chem 13: 3565-3574, 2006.

3. Hosseini SM, Samimi N, Farahmandnia M, Shakibajahromi B, Sarvestani FS, Sani M and Mohamadpour M: The Preventive effects of neural stem cells and mesenchymal stem cells intra-ventricular injection on brain stroke in rats. N Am J Med Sci 7: 390-396, 2015.

4. Tang YH, Ma YY, Zhang ZJ, Wang YT and Yang GY: Opportunities and challenges: Stem cell-based therapy for the treatment of ischemic stroke. CNS Neurosci Ther 21: 337-347, 2015.

5. Yanagisawa M and Yu RK: The expression and functions of glycoconjugates in neural stem cells. Glycobiology 17 57R-74R, 2007.

6. Park JH, Choi MR, Park KS, Kim SH, Jung KH and Chai YG: The characterization of gene expression during mouse neural stem cell differentiation in vitro. Neurosci Lett 506: 50-54, 2012.

7. Bliss T, Guzman R, Daadi M and Steinberg GK: Cell transplantation therapy for stroke. Stroke 38 (2 Suppl): 817-826, 2007.

8. Jeong CH, Kim SM, Lim JY, Ryu CH, Jun JA and Jeun SS Mesenchymal stem cells expressing brain-derived neurotrophic factor enhance endogenous neurogenesis in an ischemic stroke model. Biomed Res Int 2014: 129145, 2014.

9. Lu W, Si YI, Ding J, Chen X, Zhang X, Dong Z and Fu W: Mesenchymal stem cells attenuate acute ischemia-reperfusion injury in a rat model. Exp Ther Med 10: 2131-2137, 2015.

10. Bergström T and Forsberg-Nilsson K: Neural stem cells: Brain building blocks and beyond. Ups J Med Sci 117: 132-142, 2012

11. Trujillo CA, Schwindt TT, Martins AH, Alves JM, Mello LE and Ulrich $\mathrm{H}$ : Novel perspectives of neural stem cell differentiation: From neurotransmitters to therapeutics. Cytometry A 75: 38-53, 2009

12. Alvarez-Buylla A and Lim DA: For the long run: Maintaining germinal niches in the adult brain. Neuron 41: 683-686, 2004.
13. Taupin P: Adult neural stem cells, neurogenic niches, and cellular therapy. Stem Cells Rev 2: 213-219, 2006.

14. Benner EJ, Luciano D, Jo R, Abdi K, Paez-Gonzalez P, Sheng H, Warner DS, Liu C, Eroglu C and Kuo CT: Protective astrogenesis from the SVZ niche after injury is controlled by Notch modulator Thbs4. Nature 497: 369-373, 2013.

15. Jiao J and Chen DF: Induction of neurogenesis in nonconventional neurogenic regions of the adult central nervous system by niche astrocyte-produced signals. Stem Cells 26: 1221-1230, 2008.

16. Liu Y, Liu RR, Wang L, Zeng L, Long ZY and Wu YM: The effects of different phenotype astrocytes on neural stem cells differentiation in co-culture. Neurosci Lett 508: 61-66, 2012.

17. National Research Council of the National Academies: Guide for the care and use of laboratory animals. The National Academies Press, Washington, D.C, 2011.

18. Lü L, Luo L, Lu Y, Chen L, Xu J and Guo K: Progranulin expression in neural stem cells and their differentiated cell lineages: An immunocytochemical study. Mol Med Rep 8: 1359-1364, 2013.

19. Song H, Stevens CF and Gage FH: Astroglia induce neurogenesis from adult neural stem cells. Nature 417: 39-44, 2002.

20. Kelly S, Bliss TM, Shah AK, Sun GH, Ma M, Foo WC, Masel J, Yenari MA, Weissman IL, Uchida N, et al: Transplanted human fetal neural stem cells survive, migrate, and differentiate in ischemic rat cerebral cortex. Proc Natl Acad Sci USA 101: 11839-11844, 2004.

21. Wu Y, Wu J, Ju R, Chen Z and Xu Q: Comparison of intracerebral transplantation effects of different stem cells on rodent stroke models. Cell Biochem Funct 33: 174-182, 2015.

22. Barkho BZ, Song H, Aimone JB, Smrt RD, Kuwabara T, Nakashima K, Gage FH and Zhao X: Identification of astrocyte-expressed factors that modulate neural stem/progenitor cell differentiation. Stem Cells Dev 15: 407-421, 2006.

23. Hao L, Zou Z, Tian H, Zhang Y, Zhou H and Liu L: Stem cell-based therapies for ischemic stroke. Biomed Res Int 2014: 468748, 2014.

24. Liu Y, Wang L, Long Z, Zeng L and Wu Y: Protoplasmic astrocytes enhance the ability of neural stem cells to differentiate into neurons in vitro. PLoS One 7: e38243, 2012.

25. Nedachi T, Kawai T, Matsuwaki T, Yamanouchi K and Nishihara M: Progranulin enhances neural progenitor cell proliferation through glycogen synthase kinase $3 \beta$ phosphorylation. Neuroscience 185: 106-115, 2011. 